\title{
Pore size dependence of self-assembled type photonic crystal on dye-sensitized solar cells efficiency utilising Chlorine e6
}

\author{
George Kato $\cdot$ Chie Nishiyama $\cdot$ Takashi Yabuta $\cdot$ \\ Masahiro Miyauchi • Takuya Hashimoto • \\ Toshihiro Isobe · Akira Nakajima · Sachiko Matsushita
}

Published online: 3 December 2013

(c) The Author(s) 2013. This article is published with open access at Springerlink.com

\begin{abstract}
There are few reports on photoelectric conversion efficiency using naturally-occurring dyes for dyesensitized solar cells (DSSC). This is because the matching with an excited electronic level of naturally-occurring dye to the conduction band of semiconductor is problematic; the excited electrons are easily relaxed to the steady state with fluorescence or heat emission. We examined the fluorescence inhibition effect of a self-assembled photonic crystal using Chlorine e6 dye. Chlorine e6 is derived from chlorophyll and has a long excited electron lifetime. We prepared $\mathrm{TiO}_{2}$ inverse opals with various particle sizes by liquid phase deposition and described their effect on DSSCs with regard to structural, optical and electrochemical properties. In addition, we explored the implications of fluorescence lifetime measurements relative to the photonic band diagram and the amount of adsorbed dye. Although the main factor affecting the external photoelectric conversion efficiency was the diffusion resistance of the electrolyte and the contact resistance between $\mathrm{TiO}_{2}$ interfaces, the possibility that the dye fluorescence lifetime, i.e.
\end{abstract}

Electronic supplementary material The online version of this article (doi:10.1007/s10934-013-9761-8) contains supplementary material, which is available to authorized users.

G. Kato $\cdot$ T. Yabuta $\cdot$ M. Miyauchi $\cdot$ T. Isobe $\cdot$ A. Nakajima S. Matsushita

Department of Metallurgy and Ceramics Science, Graduate School of Science and Technology, Tokyo Institute of

Technology, 2-12-1, Ookayama, Meguro-ku, Tokyo 152-8552, Japan

G. Kato $\cdot$ C. Nishiyama $\cdot$ T. Yabuta $\cdot$ T. Hashimoto

S. Matsushita $(\varangle)$

Division of Optical and Electronic Sciences, Nihon University,

3-25-40 Sakurajosui, Setagaya-ku, Tokyo 156-8550, Japan

e-mail: matsushita.s.ab@m.titech.ac.jp the photonic band structure, can affect the internal quantum efficiency per one dye molecule was also investigated.

Keywords Graetzel cell · Inverse-opal · Colloidal crystal · Self-assembly · Self-organize

\section{Introduction}

The dye-sensitized solar cell (DSSC), first reported in 1991 [1-3], has attracted considerable attention; particularly its potential use as a next-generation solar cell. Extensive fundamental and experimental research has been conducted on DSSCs. Overall conversion efficiencies higher than $12 \%$ have been recently reported [4].

The dye might be the most important factor in achieving higher conversion efficiency. The development of effective dyes with higher light-harvesting efficiency, particularly those that work at longer wavelengths, is being actively explored [5]. Near IR absorbing dyes leading to efficiencies of more than $5 \%$ have been demonstrated in dye-sensitized solar cells [6]. However, reported dyes with high photon-to-electron conversion efficiencies are difficult to synthesise and expensive. Here we investigate how similar high-conversion efficiencies can be obtained using a naturally-occurring dye.

Electron transfer in DSSCs can be summarized as follows. The incident light at photon energy $h v_{1}$ excites the dye molecule. A large part of the excited electrons relaxes to a lower energy state and then, a certain fraction is injected into the $\mathrm{TiO}_{2}$ conduction band [7]. This results in the extraction of photocurrent from the electrode. Another fraction directly relaxes to the ground state with thermal emission and/or photon emission at photon energy $h v_{2}$, resulting in the reduction of photon-to-electron conversion efficiency. 
Characteristically, these emissions are large for naturallyoccurring dyes. This study attempts to introduce a photonic crystal structure to inhibit the emissions from the dye at $h v_{2}$ to enhance electron injection efficiency using Chlorine e6, a dye derived from chlorophyll that shows a long excited electron lifetime (the charge injection rate constant was reported as $\left.2.2 \times 10^{9} \mathrm{~s}^{-1}\right)[8,9]$ and thus is considered as an appropriate dye to examine the photonic crystal's effect.

A photonic crystal is being recognised as a promising device for incident light harvesting in DSSC research. Mallouk et al. reported a $26 \%$ improvement of light harvesting efficiency at $400-750 \mathrm{~nm}$ by combining a selfassembled photonic crystal $\left(\mathrm{TiO}_{2}\right.$ inverse opal) with DSSCs [10]. They reported that an increase in incident light path length generated by Bragg diffraction and defect scattering in the inverse opal layer resulted in the enhancement of the visible wavelength $[11,12]$.

In 2006, Miguez et al. theoretically discussed the light harvesting effect of $\mathrm{TiO}_{2}$ inverse opals in DSSCs [13, 14]. They demonstrated that the light harvesting was the result of the optical absorption amplification effect of slow photon resonant modes partially confined within the absorbing part of the cell due to the mirror behaviour of the colloidal superlattice. The effects of these defects are not discussed in this paper.

In 2010, Tao et al. described the use of a double-inverse opal as a novel optical element, which was expected to have a light scattering effect and a broader reflection region, and presented a theoretical analysis of the efficiency enhancement of designed DSSC [15]. A distinct increment of approximately $80 \%$ of photocurrent efficiency compared with a standard inverse opal layer was expected.

Not only the optical effects of $\mathrm{TiO}_{2}$ inverse opals [1619] but also the enhancement of efficient electron and electrolyte transportation generated in the inverse opals has been recently reported $[20,21]$.

In 2005, the authors reported the increase of the photonto-electron conversion efficiency per $1 \mathrm{~mol}$ of dye with an inverse opal using Chlorine e6 [22, 23]. Even though the inverse opal did not exhibit a full photonic bandgap, an increase in the spontaneous emission decay time of the dye was confirmed by time-resolved emission profiles. The authors discussed the influence of the inverse opal on the inhibition of spontaneous emissions from the dye and the possibility of the inhibition of the recombination of electrons and holes in the dye [23]. However, the discussion did not include electrochemical impedance results and the discussion was obviously insufficient.

As described above, various enhancement factors of $\mathrm{TiO}_{2}$ inverse opals in DSSCs have been reported. However, to the authors' knowledge, no report has comprehensively examined the optical effect and the electron and electrolyte transportation effects taking the photonic band diagram into consideration. Thus, we could not discuss the effect of the change in the excited electron lifetime of the dye on photon-to-electron conversion efficiency. Because the quality of the inverse opal is critical to the efficiency of DSSCs, as reported by Mallouk et al., a comprehensive examination of the same electrode is required to clarify the role of the inverse opal.

This paper presents a comprehensive examination of inverse opals of various pore sizes $(202,356,457$ and $731 \mathrm{~nm}$ diameter) using structural analysis, transmission and scattering reflection UV/Vis spectroscopy and photovoltaic, electrochemical impedance and fluorescence lifetime measurements. The paper also includes a discussion of photonic band simulation using Chlorine e6. Each analytical parameter is explored from the viewpoint of pore-size dependence. The effects of the inverse opals are summarized and a tentative analysis of the effect of fluorescence lifetime is presented.

\section{Experimental}

\subsection{Preparation}

All experiments were performed in an open air environment. Unless otherwise stated, all reagents were purchased from Waco Pure Chemical Industries, Ltd.

\subsubsection{Preparation of polystyrene opals}

Indium thin oxide (ITO) substrates $(10 \Omega / \square, 2 \mathrm{~cm} \times 3 \mathrm{~cm}$, thickness $2,000 \pm 200 \AA$, Geomatec Co.) were cleaned by sonication for $20 \mathrm{~min}$ in pure water, and subsequently for $20 \mathrm{~min}$ in ethanol. Then, the ITO substrates were rinsed with pure water and air dried. Polystyrene opals of each size were prepared by an evaporation-driven self-assembly method. The details of this method have been previously reported [24, 25]. Polystyrene particle suspensions (of diameters 202, 356, 457 and $731 \mathrm{~nm}$ with $2.62,2.6,2.5$ and $2.63 \%$ solids in water, respectively, Polysciences, Inc.) were sonicated for $5 \mathrm{~min}$. The polystyrene particle suspensions were injected in a glass cell and coated onto the ITO substrate. The opals were kept at $60{ }^{\circ} \mathrm{C}$ for $30 \mathrm{~min}$. The ITO substrate was set on an $x$ stage and moved at a constant speed of $2 \mu \mathrm{m} / \mathrm{s}$ (USJ Co.). The polystyrene opals were dried at $60{ }^{\circ} \mathrm{C}$ for $30 \mathrm{~min}$. The polystyrene opals composed of 202, 356, 457 and 731-nm diameter particles have been referred as PS202, PS356, PS457 and PS731, respectively.

\subsubsection{Preparation of $\mathrm{TiO}_{2}$ inverse opal electrodes}

The authors had assessed the structural analysis, photovoltaic characteristics and electrochemical impedance 
measurements of the normal and inverse opal dye-sensitized electrodes prepared by three different methods: electrophoretic deposition, $\mathrm{TiCl}_{4}$ coating and liquid-phase deposition (LPD) [26]. The electrode prepared by LPD exhibited the least internal resistance and highest photonto-electron conversion efficiency among the three methods. Therefore, in this study, $\mathrm{TiO}_{2}$ inverse opals were synthesised by LPD. The samples were immersed in a solution of $0.15 \%$ titanium isopropoxide $\left(\left[\left(\mathrm{CH}_{3}\right)_{2} \mathrm{ChO}\right]_{4} \mathrm{Ti}, 95.0 \%\right)$ and nitric acid in ethanol (Junsei Chemical Co., Ltd.) for $5 \mathrm{~min}$ at room temperature. The samples were dried and immersed in a solution of $0.1 \mathrm{M}$ of ammonium hexafluorotitanate $\left(\left(\mathrm{NH}_{4}\right)_{2} \mathrm{TiF}_{6}\right)$ and $0.2 \mathrm{M}$ of boric acid in pure water for $20 \mathrm{~min}$ at $60^{\circ} \mathrm{C}$. The $\mathrm{pH}$ of the solution was adjusted to 3.0 using hydrochloric acid. The samples were rinsed in pure water and dried for several hours. The samples were heated in air at $0.55{ }^{\circ} \mathrm{C} / \mathrm{min}$ to $500{ }^{\circ} \mathrm{C}$ for $30 \mathrm{~min}$ and cooled to room temperature. The inverse opals prepared using PS202, PS356, PS457 and PS731 have been referred as IO202, IO356, IO457 and IO731, respectively. An ITO substrate without opal structure was also processed by LPD for use as a reference electrode (referred as $\mathrm{TiO}_{2^{-}}$ coated ITO).

\subsubsection{Electrode assembly}

$\mathrm{TiO}_{2}$ inverse opal electrodes thus prepared were heated to $200{ }^{\circ} \mathrm{C}$ for $10 \mathrm{~min}$ using a hot plate (HP-400A, Azuwan $\mathrm{Co}$ ). The electrodes were immersed in a $0.3-\mathrm{mM}$ solution of Chlorine e6 trisodium salt (Fig. 1, Tama Biochemical Co.)/chenodeoxycholic acid methanol solution at $40{ }^{\circ} \mathrm{C}$ for $24 \mathrm{~h}$. After sensitization, the active area of the $\mathrm{TiO}_{2}$ inverse opals was reduced to $0.25 \mathrm{~cm}^{2}$ by scrapping away excess material. Parafilm $\left(0.25 \mathrm{~cm}^{2}\right)$ was used as a spacer between the $\mathrm{TiO}_{2}$ inverse opal electrodes and platinized ITO $(2 \mathrm{~cm} \times 3 \mathrm{~cm})$. An electrolyte $\left(0.05 \mathrm{M} \mathrm{I}_{2} / 0.1 \mathrm{M} \mathrm{LiI} / 0.5 \mathrm{M}\right.$ 4-tert-Butylpyridine (Tokyo Chemical Industry Co., Ltd)/ 0.6 M DMPII (1,2-dimethyl-3- propylimidazolium iodide, Solaronix) in acetonitrile) was dropped on the active area. The dye-sensitized electrodes and the platinized ITO substrates were sandwiched with binder clips.

\subsection{Material analysis}

The electrodes were observed using an optical microscope (BX60, OLYMPUS) and scanning electron microscope (SEM, VE9800SP (Keyence Co.) and S4500 (Hitachi, Ltd.)). The X-ray diffraction (XRD) spectra of the inverse opals were obtained using an X-ray diffractometer $(50 \mathrm{kV}$, $250 \mathrm{~mA}$, RINT2500 VPC, Rigaku) equipped with a graphite monochromator using $\mathrm{Cu} K \alpha$ line $(\lambda=1.54 \AA)$ at room temperature. The results were compared with the database of the Joint Committee on Powder Diffraction

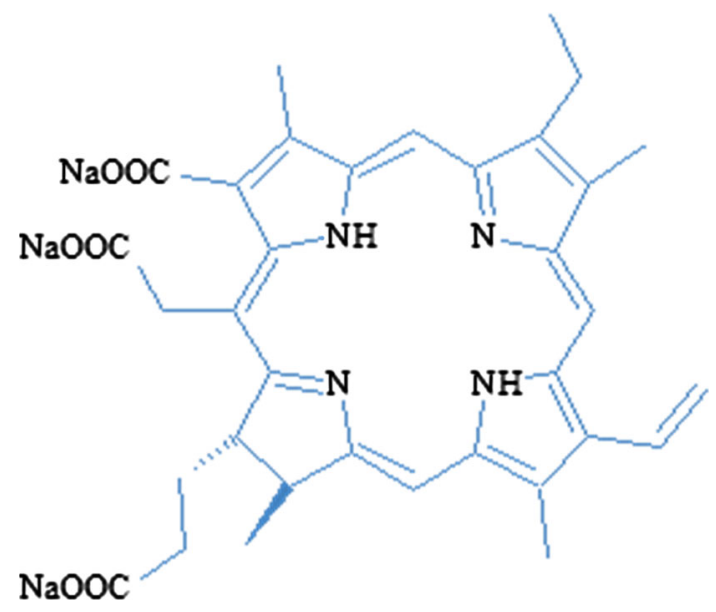

Fig. 1 Chemical structure of Chlorine e6 trisodium salt

Standards [27]. The thickness of the inverse opal layers was measured with a laser microscope (OLS4000, OLYMPUS). Transmittance spectra were collected using an UV/Vis spectrophotometer (UV650, Jasco). Diffuse reflectance spectra were measured using a spectrometer equipped with an integrating sphere attachment (ISV-722, Jasco).

\subsection{Photonic band simulation}

Photonic bands were determined by photonic band calculation software (BandSOLVE, Rsoft Design Group). The face-centred cubic (fcc) packing structures of the polystyrene $(n=1.55)$ sphere in air $(n=1.00)$ and the air sphere in $\mathrm{TiO}_{2}(n=2.54)$ were calculated. Each path of Brillouin zone was divided by 9. A Windows computer (Dell Dimension 8300) was used.

\subsection{Photoelectrochemical analysis}

\subsubsection{I-V measurement}

The cell was connected to a potentiostat (HSV-100, Hokuto Denko Corporation) for $I-V$ curve measurements. These measurements were obtained with light (AM 1.5) from a solar simulator (Jasco) on the ITO substrate side of the cell. The $I-V$ curve measurement was conducted from 0.8 to $-0.1 \mathrm{~V}(100 \mathrm{mV} / \mathrm{s})$ and was controlled by a potentiostat. The electrode area was $0.25 \mathrm{~cm}^{2}$.

\subsubsection{Electrochemical impedance measurement}

The electrochemical impedance spectroscopic (EIS) analysis was performed using a VersaSTAT 3 electrochemical impedance system (Toyo) by applying a $10-\mathrm{mV}$ amplitude 
signal in the frequency range of $1-10,000 \mathrm{~Hz}$. In the EIS measurements, the electrodes were same as those for the $I-V$ measurement.

\subsubsection{Measurement of the amount of adsorbed dye molecules}

To spectrophotometrically evaluate the amount of adsorbed dye molecules on the electrodes, the dye on a known electrode area was detached by immersion for $5 \mathrm{~h}$ in a 0.1 M NaOH $\mathrm{H}_{2} \mathrm{O} / \mathrm{EtOH}$ (50:50) solution [14] and the absorption intensity of the resulting dye solution was measured using a UV/Vis spectrophotometer (JASCO, V650). The measured intensity was converted into the concentration of adsorbed dye molecules.

\subsubsection{Incident photon-to-current conversion efficiency (IPCE) measurement}

The IPCE spectra were measured as a function of wavelength from 400 to $800 \mathrm{~nm}$ using an IPCE system (SM250, Bunkoukeiki), particularly designed for DSSCs. The light intensity was $5 \mathrm{~mW} / \mathrm{cm}^{2}$.

\subsubsection{Measurement of fluorescence lifetime spectra}

The fluorescence lifetime on the $\mathrm{TiO}_{2}$ electrodes was determined by transient fluorescence spectra (C7700-ABS$\mathrm{N}$, Hamamatsu) particularly designed for DSSCs. A sample was excited using nanosecond optical pulses (4 ns width and $532 \mathrm{~nm}$ wavelength), generated using a Nd:YAG laser. The number of scans was 3000 .

\section{Results and discussion}

To discuss this complicated phenomena, firstly, we would like to show our inverse opals quality in Sect. 3.1. After showing our inverse opal's structure, the optical measurement spectra were shown in Sects. 3.2 and 3.3, and compared with the calculated photonic band diagram. The solar cell's characteristics using these measured inverse opals were introduced in Sects. 3.4-3.7. The fluorescence lifetime was reported in Sect. 3.8, and all results were discussed in the following Conclusion.

\subsection{Quality of polystyrene opals and inverse opals}

The visual images of prepared polystyrene opals are shown in Fig. 2. Each polystyrene opal shows structural colours, i.e. blue, green, red and white for PS202, PS356, PS457 and PS731, respectively. The white colour shown in PS731 might be caused by Mie scattering because the diameter of PS731 is larger than the visible wavelength. The number of layers of these opals was almost same.

The exfoliation of the opals was observed when they were dipped in the titanium solution for LPD. In particular, PS202 and PS457 were easily exfoliated from the substrate and formed microfibres during the subsequent drying process [28]. The aqueous particle suspensions had minimal surfactant; therefore, the surfactant effect on the exfoliation could be ignored. It is considered that the fibre structure was formed by the shrinking of particles' homogeneous films such as PS202 and PS457. The improvement of the coalescent process would prevent the exfoliation of the opals.

A large number of cracks were generated on the surface of PS202 and PS457 during the drying phase of the LPD
Fig. 2 Optical microscopic images of opals fabricated from 202 nm- (a), 356 nm- (b), $457 \mathrm{~nm}-(\mathbf{c})$ and $731 \mathrm{~nm}-$ (d) PS spheres. Each scale bar indicates $5 \mathrm{~mm}$
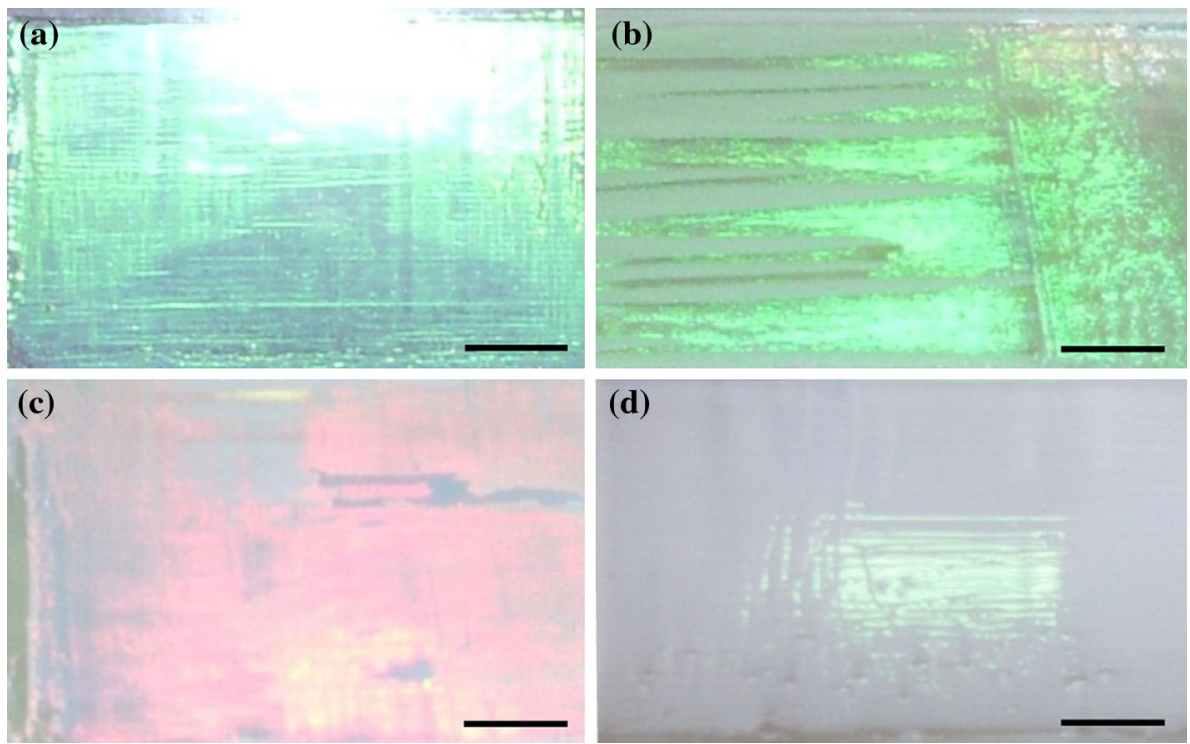
Fig. 3 SEM images of inverse opals fabricated from $202 \mathrm{~nm}$ (a), $356 \mathrm{~nm}-$ (b), $457 \mathrm{~nm}$ -

(c) and $731 \mathrm{~nm}$ - (d) PS spheres
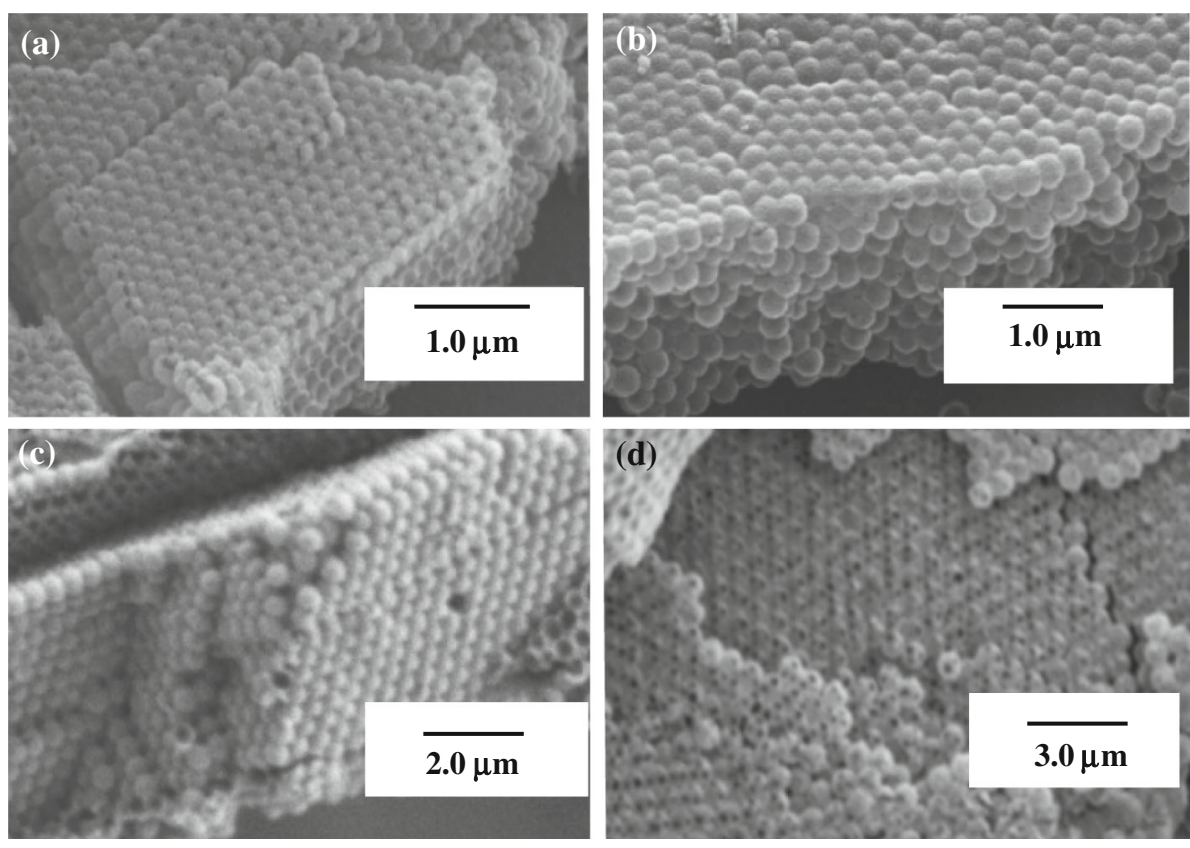

seeding step. The reason of these cracks might be also the shrinking of particles' homogeneous films.

After sintering, the samples formed inverse opal structures that reflected each original opal's structure (Fig. 3). It is clear that the polystyrene particles we used were dense enough to fabricate our inverse opals. The SEM images revealed that the diameters of $\mathrm{TiO}_{2}$ inverse opals were $10 \%$ less than those of original opals; the diameters of IO202, IO356, IO457 and IO731 became 180, 330, 400 and $680 \mathrm{~nm}$, respectively. It is assumed that the shrinkage was caused by the change in polystyrene particles' volume during calcination, as often reported [29]. The SEM images also revealed that our inverse opals have some defects. It is reported that, even though the opals could not avoid from defects, the change of photon group velocity was observed if the periods was over 10 [30].

The XRD patterns in Fig. 4 indicate that all inverse opals were in anatase phase. The LPD deposition used in this manuscript was developed by Deki et al. [31]. The total reaction scheme is:

$$
\begin{aligned}
& {\left[\mathrm{TiF}_{6}\right]^{2-}+\mathrm{nH}_{2} \mathrm{O}=\left[\mathrm{TiF}_{6-\mathrm{n}}(\mathrm{OH})_{\mathrm{n}}\right]^{2-}+\mathrm{nHF}} \\
& \mathrm{H}_{3} \mathrm{BO}_{3}+4 \mathrm{HF} \rightarrow \mathrm{HBF}_{4}+3 \mathrm{H}_{2} \mathrm{O}
\end{aligned}
$$

Thus, boron doping into $\mathrm{TiO}_{2}$ should be negligible. Regarding with the fluorine doping, Deki et al. had reported that fluorine was not detected by the qualitative analysis using $\mathrm{CaCl}_{2}$ for the samples which dissolved in diluted $\mathrm{HNO}_{3}$.

The thickness of the $\mathrm{TiO}_{2}$ layers is shown in Table 1 . The amount of dye in the $\mathrm{TiO}_{2}$ layer will build up with increasing film thickness. It has been reported that generally the photocurrent increases with the thickness;

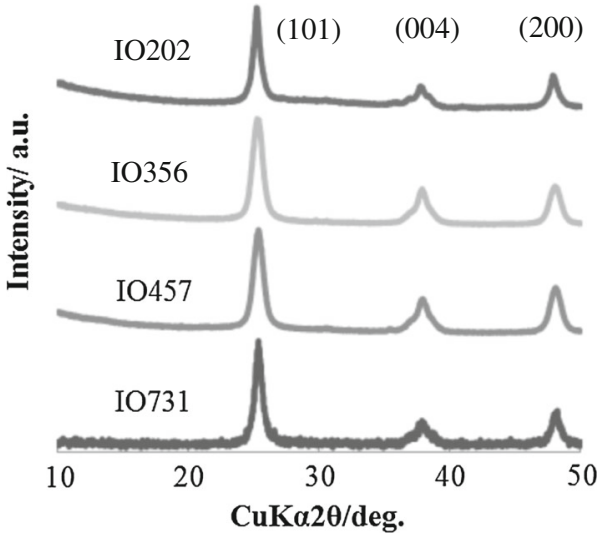

Fig. 4 XRD pattern of inverse opals fabricated from $202 \mathrm{~nm}$-, $356 \mathrm{~nm}-, 457 \mathrm{~nm}-$ and $731 \mathrm{~nm}-\mathrm{PS}$ spheres

however, when film thickness is greater than approximately $20 \mu \mathrm{m}$, the photocurrent decreases [32, 33]. It is considered that the charge recombination becomes more severe in films thicker than $20 \mu \mathrm{m}[34,35]$. In our case, the thicknesses were sufficient to examine the photonic crystal effect and were almost same for each inverse opal. This result showed that there was no need to consider the difference of thickness.

\subsection{Incident angle dependence of transmission spectrum}

Supporting Information 1 shows the incident angle dependence transmission spectra of the polystyrene opal structure. Deep transmittance dips shifting with incident angle were observed in PS202 and PS356. These shifts 
Table 1 Thickness of the $\mathrm{TiO}_{2}$ layers

\begin{tabular}{|c|c|}
\hline $\mathrm{TiO}_{2}$ layer & Thickness/ $\mu \mathrm{m}$ \\
\hline $\mathrm{TiO}_{2}$-coated ITO & $5.7 \pm 0.3$ \\
\hline IO202 & $7.1 \pm 0.1$ \\
\hline IO356 & $6.6 \pm 0.3$ \\
\hline IO457 & $6.2 \pm 0.1$ \\
\hline IO731 & $6.1 \pm 0.1$ \\
\hline
\end{tabular}

were caused by Bragg diffraction. The theoretical wavelength of the first-order Bragg diffraction peak can be simulated using the average refractive index, as shown in Eq. (1):

$\lambda_{\text {max }}=2 d \sqrt{n_{s}^{2} f+n_{v}^{2}(1-f)}$

where $\lambda_{\max }$ is the transmittance dip wavelength, $n_{s}$ is the refractive index of polystyrene (1.38), $n_{v}$ is the refractive index of air (1.0), $f$ is the filling fraction of polystyrene (ca. 0.74 for fcc packing) and $d$ is the period of the structure (ca. $0.81 \times$ particle diameter for our structure). The calculated transmittance dips according to Eq. (1) were 453, 800, 1,026 and $1643 \mathrm{~nm}$ for PS202, PS356, PS457 and PS731, respectively.

The experimental transmittance dip wavelengths of PS202 and PS356 fit well with the calculated values. No large dips were observed in the transmittance spectra of PS457 and PS731 because the expected dips were positioned out of our evaluation range. Here we should mention that, in PS457, the transmittance dips around $500 \mathrm{~nm}$ showed incident angle dependence that is a specific characteristic of Bragg diffraction. We consider that these dips around $500 \mathrm{~nm}$ were due to second-order Bragg diffraction.

The incident angle dependence of the transmission spectra of $\mathrm{TiO}_{2}$ inverse opals is shown in Supporting Information 2. The dips of the spectra of $\mathrm{TiO}_{2}$ inverse opals were less sharp than those of the original opals. This lack of sharpness may be due to periodical disorder. $\mathrm{TiO}_{2}$ coated ITO showed no specific dips in the measurement range (Supporting Information 2a). The observed transmission dips around 500 and $550 \mathrm{~nm}$ for IO202 and IO356, respectively, were shifted by the incident angles. This result showed that these dip were caused by Bragg diffraction. Similar to the transmittance spectra of PS457 and PS731, no transmission dip were observed for IO457 and IO731 because the expected dips were also positioned out of our evaluation range. The transmission dips of the inverse opals became smaller than those of the original opals, probably because of the increase of defects created during the LPD process.
3.3 Diffuse reflectance spectra and photonic band diagrams

The diffuse reflectance spectra of the original opals and unsensitised $\mathrm{TiO}_{2}$ inverse opals are shown in Fig. 5. The diffuse reflectance spectra include the experimental reflectance from all directions of the substrate; therefore, a comparison with calculated photonic band diagrams is possible. The reflectance peaks related to the periodic structures were observed for each inverse opal structure but were not observed for $\mathrm{TiO}_{2}$-coated ITO substrates.

As shown in Fig. 5a, sharp reflectance peaks were observed for PS202, PS356 and PS457. In general, opals prepared under gravity form fcc structures. The SEM images (Fig. 3) of inverse opals suggest that our original opals were a fcc arrangement of monodispersed polystyrene spheres with the close-packed plane (111) oriented parallel to the underlying glass substrate. Thus, the diffuse reflectance spectra represented all reflected light from the (111) plane, i.e. the photonic bands along the $\Gamma-L$ and $L-X$ directions. The diffuse reflectance peak was positioned around a quasi bandgap in the photonic band diagram as shown in broken arrows (Fig. 5c). The parameters for the photonic band diagram are the refractive indices and the normalized frequency, that is defined as (the periodicity of the structure a)/(the light wavelength $\lambda$ ). Thus, we can compare each structure in the same photonic band diagram. The experimental result is in good agreement with the calculation. The low transmittance of PS731 in the overall wavelength measurement might be due to Mie scattering as previously discussed in the section on the quality of polystyrene opals and inverse opals.

As shown in Fig. 5b, the lack of sharpness of the diffuse reflectance spectra might be due to periodical disorder, as discussed in the previous section. Miyazaki and Ohtaka had revealed that, even in two-dimensional colloidal crystal composed of polyvinyltoluene particles (the refractive index $=1.6$ ), the spectrum for the colloidal crystal with 6 periods had well-developed dips and was similar to the calculated result for an infinite lattice [30]. The inverse opal has a larger photon confinement efficiency than the opal has, and, our inverse opal showed more than 10 layers in one domain. We believe that our comparison between theoretically obtained photonic band diagrams and experimental data give us valid knowledge. The normalized frequency was calculated by the diameters of the air sphere of inverse opals, i.e. smaller diameters than the original polystyrene particle diameters, as shown in Fig. 5d. Since our inverse opals are composed of $\mathrm{TiO}_{2}$, incident light less than $400 \mathrm{~nm}$ wavelength are absorbed. Thus we could not compare our experimental spectra with our calculated photonic band diagram with the view point of photonic crystal, and we decided not to show our experimental 
Fig. 5 Diffusion reflectance spectra $(\mathbf{a}, \mathbf{b})$, photonic band diagrams and normalized diffusion reflectance spectra (c, d) of opals (a, c) and inverse opals $(\mathbf{b}, \mathbf{d})$ fabricated from $202 \mathrm{~nm}$ - (grey line), $356 \mathrm{~nm}$ (grey dotted line), $457 \mathrm{~nm}$ (black line) and $731 \mathrm{nm-(black}$ dotted line) PS spheres. The spectra of ITO and $\mathrm{TiO}_{2}$-coated ITO (grey dashed line) are also shown in $\mathbf{a}, \mathbf{b}$. The fcc Brillouin zone is shown in the inset (a)
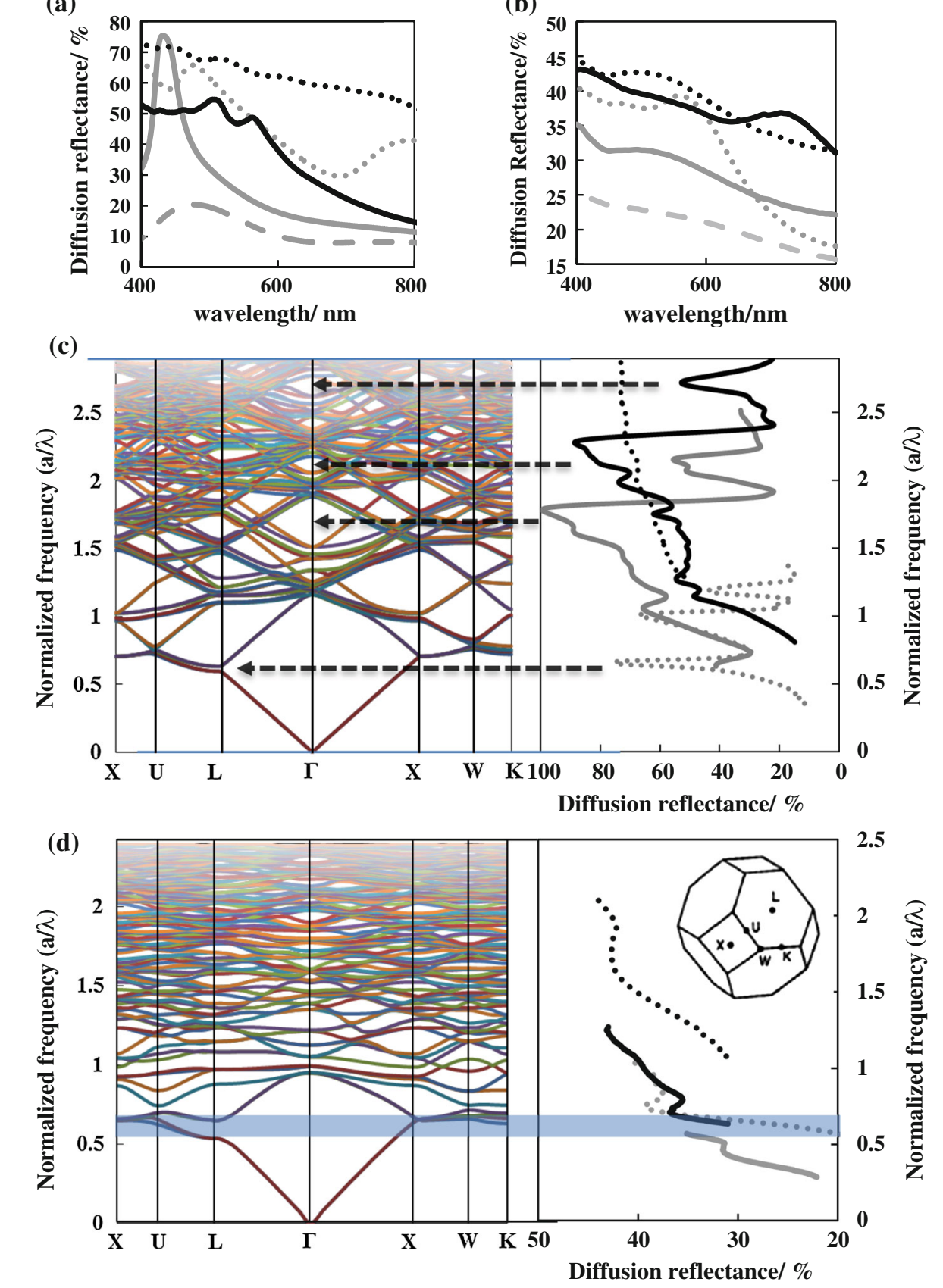

(b)

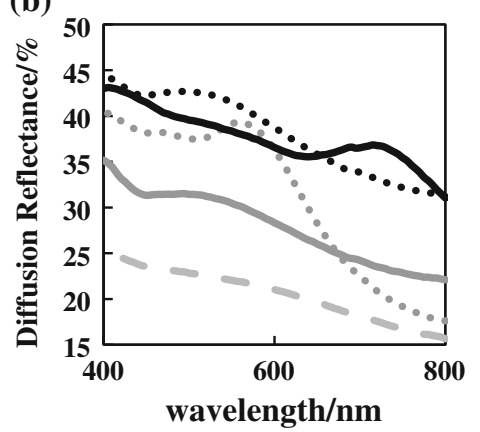

spectra less than $420 \mathrm{~nm}$ in Fig. 5d. The diffuse reflectance dips of IO356 and IO457 at 0.7 (showed as a translucent square in Fig. 5d) are in good agreement with the calculated photonic bandgap at $\Gamma$. The reflectance peak of IO202 was at a lower frequency than those of IO356 and IO431. This may be due to a large component of air in the structure produced by the particle exfoliation, as previously described in the section on polystyrene and inverse opal quality that resulted in an average refractive index lower than the estimated value. As a result, the reflectance peak may have been observed on the normalized frequency lower than the calculated value. For IO731, the observed broad peak reflected the quasi gap near $\Gamma$ point.

From the above results, it can be said that our inverse opals' periodicity was sufficient for a photonic crystal in this observation wavelength range.

\section{4 $I-V$ measurement}

The $\mathrm{TiO}_{2}$ layer has an important role as an electron transportation medium [36-39]. The $I-V$ curve of $\mathrm{TiO}_{2}$ inverse opals with various particle diameters is shown in Fig. $6 \mathrm{a}$, and 
Fig. 6 I-V curve (a), IPCE spectra (b), internal IPCE spectra (c) and normalized internal IPCE spectra by the amount of adsorbed dye (d) of inverse opals fabricated from $202 \mathrm{~nm}$ - (grey line), $356 \mathrm{~nm}$ (grey dotted line), $457 \mathrm{~nm}$ (black line) and $731 \mathrm{~nm}$-(black dotted line) PS spheres. The spectra of $\mathrm{TiO}_{2}$-coated ITO (grey dashed line) are also shown
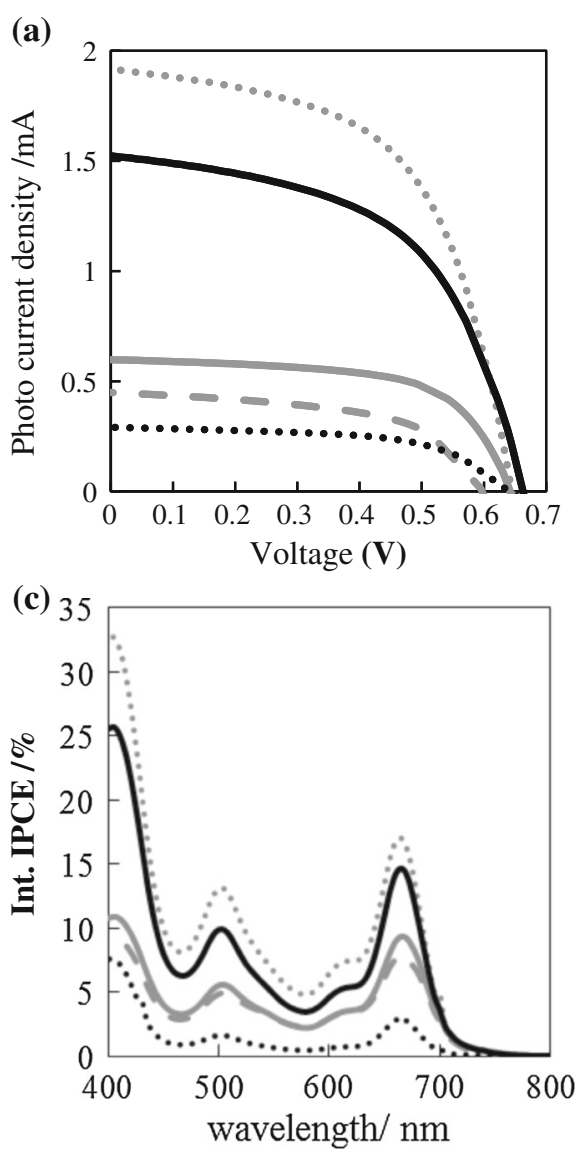
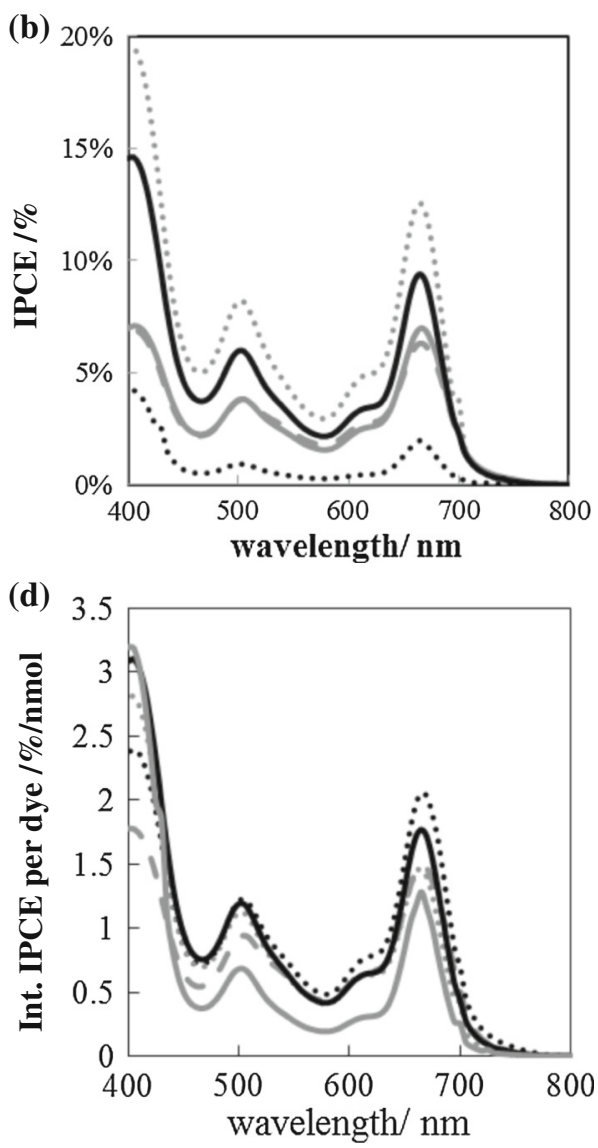

the solar cell characteristics are shown in Table 2. Here Chlorine e6 derived from chlorophyll origin dye and having a long fluorescence lifetime was used. A photoelectric conversion efficiency of $0.4 \%$ has been reported for a system that used Chlorine e6 in a DSSC cell with P25 and an iodinebased electrolyte [40]. Photoelectric conversion efficiencies that exceeded those reported were attained with IO356 and IO457. $J_{s c}$ became maximum in IO356 and decreased as the particle diameter increased. The absorption wavelength range of Chlorine e 6 is $400-700 \mathrm{~nm}$. The integration value of the diffuse reflectance at this range increased as the particle diameter increased, as shown in Fig. 5b. This larger reflection of incident light may cause the $\mathrm{J}_{\mathrm{sc}}$ to become small. The $J_{s c}$ is also discussed in relation to the amount of dye adsorption and an electrochemistry impedance result in the latter sections. The relationship between $V_{o c}$ and the size of pores in inverse opals was not convincing. In general, it is supposed that $V_{o c}$ is dependent on the constituent materials in a battery. As shown in Fig. 4, the inverse opals that we produced were almost electrochemically homogeneous anatase. The increase of F.F. was almost proportional to the increase in the diameter of the pores in the inverse opals. Although our results do not provide sufficient evidence to confidently assert that the size of the pores is a determining factor for F.F., it is supposed that F.F. will become so small
Table 2 Photovoltaic parameters of dye-sensitized solar cells containing $\mathrm{TiO}_{2}$ layers

\begin{tabular}{lllllll}
\hline $\begin{array}{l}\mathrm{TiO}_{2} \\
\text { layer }\end{array}$ & $\begin{array}{l}\mathrm{J}_{\mathrm{sc}} \\
(\mathrm{mA} / \\
\left.\mathrm{cm}^{2}\right)\end{array}$ & $\begin{array}{l}\mathrm{V}_{\mathrm{oc}} \\
(\mathrm{V})\end{array}$ & F.F. & $\begin{array}{l}\eta \\
(\%)\end{array}$ & $\begin{array}{l}\text { Amount of } \\
\text { adsorbed dye } \\
(\mathrm{nmol})\end{array}$ & $\begin{array}{l}\text { Normalized } \\
\eta(\% / \mathrm{nmol})\end{array}$ \\
\hline $\begin{array}{c}\mathrm{TiO}_{2^{-}} \\
\text {coated }\end{array}$ & 0.45 & 0.64 & 0.52 & 0.14 & 2.13 & 0.066 \\
$\mathrm{ITO}$ & & & & & & \\
$\mathrm{IO} 202$ & 0.68 & 0.68 & 0.55 & 0.28 & 4.37 & 0.064 \\
$\mathrm{IO} 356$ & 1.35 & 0.65 & 0.58 & 0.58 & 5.17 & 0.112 \\
$\mathrm{IO} 457$ & 1.16 & 0.66 & 0.6 & 0.43 & 6.32 & 0.068 \\
$\mathrm{IO} 731$ & 0.26 & 0.65 & 0.61 & 0.10 & 4.13 & 0.024 \\
\hline
\end{tabular}

that the resistance component in a conductive substrate will be large. In our case, the interaction of a substrate and a $\mathrm{TiO}_{2}$ inverse opal may have contributed to the improvement in the $F$.F. This will be discussed in relation to the electrochemistry impedance measurement results presented below.

\subsection{Electrochemical impedance analysis}

The Nyquist plots of various $\mathrm{TiO}_{2}$ inverse opals are presented in Fig. 7a. The total resistance of our system was larger than that reported by other researchers because we 

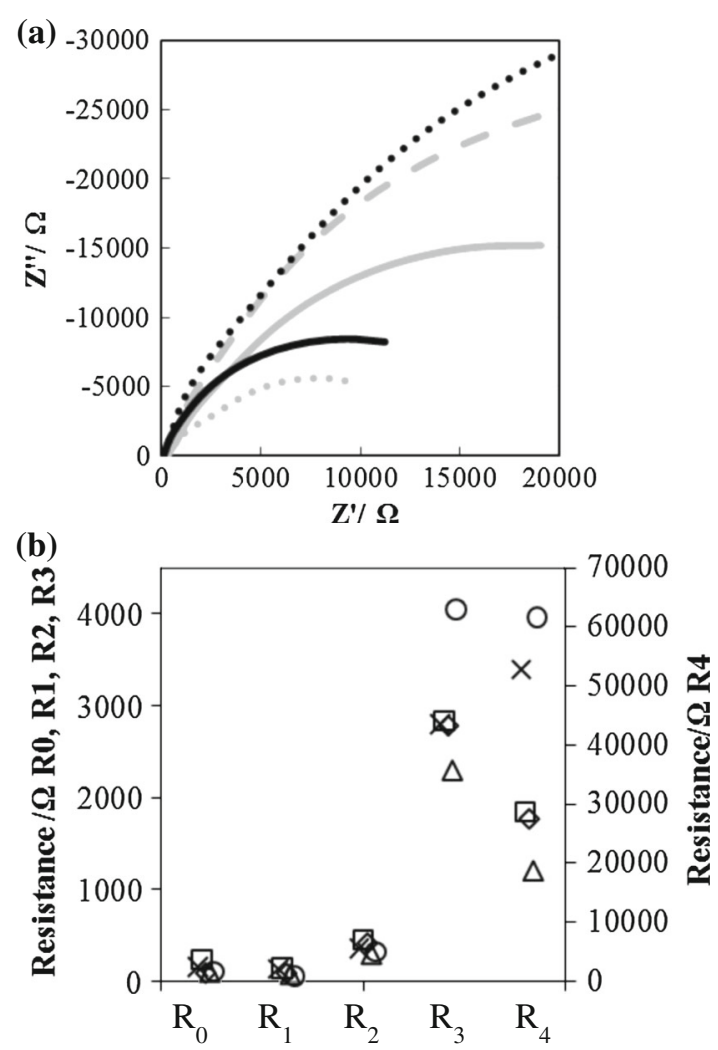

Fig. 7 Cole-cole plots (a) and resistance elements (b) of IO202 (grey line, square), IO356 (grey dotted line, triangle), IO457 (black line, diamond) and $\mathrm{IO} 731$ (black dotted line, circle). The spectra of $\mathrm{TiO}_{2^{-}}$ coated ITO (grey dashed line, times symbol) are also shown. $\mathrm{R}_{0}$, resistance at substrate; $\mathrm{R}_{1}$, resistance at substrate/TiO $\mathrm{T}_{2}$ interface; $\mathrm{R}_{2}$, resistance between $\mathrm{TiO}_{2}$ particles; $\mathrm{R}_{3}$, resistance at Pt/electrolyte interface and electrolyte/dye/ $/ \mathrm{TiO}_{2}$ interface; $\mathrm{R}_{4}$, diffusion resistance of the electrolyte

used Chlorine e6. The results were fitted to the DSSC circuit model and each resistance component was analysed (Fig. 7b). The resistance component of a DSSC consists of two or more factors such as an ITO substrate, dye, $\mathrm{TiO}_{2}$ and an electrolyte solution. The various applications of electrochemical impedance measurements to DSSCs have been performed and there are also numerous reports of equivalent circuits for the separation of the resistive component [41-43]. In this experiment, we used the equivalent circuit reported by Eguchi et al. [44], which enables each resistance component of DSSCs to be discussed in detail. Five resistance components are considered. $\mathrm{R}_{0}$ is the resistance of the ITO substrate. $R_{1}$ at $10^{4}-10^{3} \mathrm{~Hz}$ is the resistance of the substrate $/ \mathrm{TiO}_{2}$ interface. $\mathrm{R}_{2}$ at $10^{3}-10^{2} \mathrm{~Hz}$ is the contact resistance between $\mathrm{TiO}_{2}$ interfaces. $\mathrm{R}_{3}$ at $10^{1}$ $\mathrm{Hz}$ is the resistance of the Pt/electrolyte interface and the electrolyte/dye $/ \mathrm{TiO}_{2}$ interface. $\mathrm{R}_{4}$ at $10^{0} \mathrm{~Hz}$ is the diffusion resistance of the electrolyte. Here we should mention that it was not easy to separate $R_{3}$ and $R_{4}$ in our system.
$\mathrm{R}_{1}$ and $\mathrm{R}_{2}$ became smaller in particles with larger diameters. This trend might be because of the wider contact area between $\mathrm{TiO}_{2}$ and the substrate for $\mathrm{R}_{1}$ and $\mathrm{TiO}_{2}$ particles for $R_{2}$ in particles with larger pore sizes. $R_{3}$ and $R_{4}$ became the smallest in IO356. In general, an inverse opal is considered to stimulate the diffusion of the electrolyte solution. In IO356, it is considered that electron injection into titanium oxide is promoted at the $\mathrm{TiO}_{2} /$ electrolyte interface, probably because of the larger interface area, and leads to the decline of $R_{4}$ and $R_{3}$. A possible reason for large $R_{3}$ and $R_{4}$ in IO731 is that the thicker shell of IO731 prevents diffusion of the electrolyte inside the pores, as can be seen from the SEM images presented in Fig. 3 .

These impedance results can elucidate the general characteristics of a solar cell in relation to pore size. It is considered that the increase of $F . F$. is due to the $\mathrm{R}_{2}$ and $\mathrm{R}_{3}$ values. This is because these resistances also increased in relation to pore size. IO356 showed the maximum $J_{s c}$ in the system because IO356 had the lowest resistance of $R_{3}$ and $\mathrm{R}_{4}$. The following sections will elaborate on these electrochemical results.

\subsection{Measurement of the dye adsorption amount}

Table 2 shows the estimated amount of dye adsorbed on various $\mathrm{TiO}_{2}$ layers. Because these inverse opals have approximately the same film thickness, when particle diameter increases, the quantity of $\mathrm{TiO}_{2}$ decreases, i.e. dye adsorption sites should decrease. However, in our experiment, IO457 showed the largest amount of adsorbed dye. The adsorption of the dye was performed by soaking the inverse opals in an ethanol solution overnight. It is unlikely that the dye ethanol solution did not permeate pores larger than $180 \mathrm{~nm}$. The Pore size was probably not a dependent factor. It is more likely that the dye solution did not enter the pores because of the thickness of the walls of inverse opals. This conclusion is supported by the large $\mathrm{R}_{4}$ in IO731.

\subsection{Incident photon-to-electron conversion efficiency}

Figure $7 \mathrm{~b}$ shows the IPCE results of various $\mathrm{TiO}_{2}$ inverse opals. The photoelectric conversion efficiency is the lowest in IO731 and highest in IO356. These IPCE results are consistent with the results of the $I-V$ measurements and include the effect of light reflected from the surface of the DSSC. Thus, the internal quantum efficiency was determined by the diffuse reflectance spectra (Fig. 6c).

The internal quantum efficiency at $670 \mathrm{~nm}$ of IO202 and IO356 was largely declined compared with those of other electrodes. This result confirmed that the diffuse reflectance of the long wavelength region of IO202 and IO356 was low (Fig. 5). The overall trend due to changes in 
particle size was almost same for the external quantum efficiency.

The internal quantum efficiency per one dye molecule, i.e. the internal quantum efficiency divided by the amount of adsorbed dye, is shown in Fig. 6d. Unlike other IPCE spectra (Fig. 6b, c), the particle size that indicates maximum efficiency was different with different incident wavelengths. The absorption peaks of $400 \mathrm{~nm}$ in IO731, $500 \mathrm{~nm}$ in IO202 and IO457 and $670 \mathrm{~nm}$ in IO202 show maximum efficiency. In addition, the internal quantum efficiency of IO202 was less than $\mathrm{TiO}_{2}$-coated ITO at wavelengths above $450 \mathrm{~nm}$. As the reason of this incident wavelength dependence of internal quantum efficiency per one dye molecule, the scattering effect of incident light in the inverse opal layer and/or any changes in electron injection efficiency from the dye to titanium oxide caused by any specific effect were conceivable.

First, we consider the scattering effect of incident light. Mie theory [45, 46] and Anderson localisation of light [47] can analytically describe the scattering of light by spherical particles. Ferber et al. [48] simulated light scattering effects in DSSC using Mie theory and the radiative transfer equation. Effective Mie scatters are observed for particles with sizes in the range of visible light wavelengths. In other words, the larger scattering intensity of long wavelengths was observed in larger particles. In our results, the internal quantum efficiency per mole of dye with small particle size was improved for longer incident light wavelengths, i.e. the scattering effect of incident light was not the cause of this incident wavelength dependence. Rayleigh scattering that is adapted to fine particles in light scattering is also relevant. It is thought that the primary particle diameter of titanium oxide that constitutes an inverse opal did not significantly change among our inverse opals. Therefore, Rayleigh scattering is also not considered to be the cause of this incident wavelength dependence. It is possible that the electron injection efficiency from the dye to titanium oxide was changed for any reason as discussed below.

\subsection{Fluorescence lifetime measurement}

To explain the incident light wavelength dependency, fluorescence lifetime measurement was performed. The wavelength for the measurement of fluorescence lifetime was set to $675 \mathrm{~nm}$, which is the wavelength of maximum emission of Chlorine e6. The photocurrent was not increased during this measurement. If the fluorescence of the dye was influenced by a particular characteristic of the photonic crystal, the extension of fluorescence lifetime at $675 \mathrm{~nm}$ should be evident. The sensitive dependencies of the radiative lifetime of CdTe quantum dots [49] and Rhodamine 6G [50] have already demonstrated the effect of the quasi photonic bandgap on the spontaneous emission
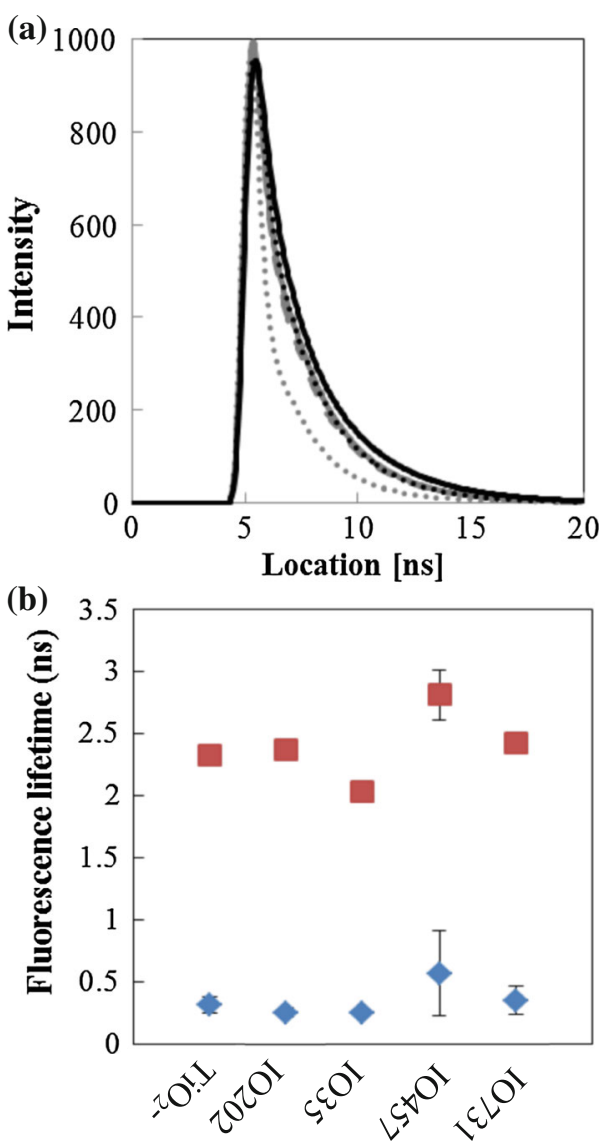

Fig. 8 Time-resolved emission profiles (a) and change in fluorescence lifetime constant (b) of $\tau_{1}$ (diamond) and $\tau_{2}$ (square) components of dye-sensitized electrodes fabricated from IO202 (grey line), IO356 (grey dotted line), IO457 (black line) and IO731 (black dotted line). The spectra of $\mathrm{TiO}_{2}$-coated ITO (grey dashed line) are also shown. The error bars show statistical standard errors

in self-assembled photonic crystals. The fluorescence decay curve is shown in Fig. 8a. The fluorescence lifetime was divided into a short life component $\left(\tau_{1}\right)$ and longer life component $\left(\tau_{2}\right)$. Previous research has revealed that $\tau_{1}$ (approximately $0.4 \mathrm{~ns}$ ) was the component injected into the conduction band of $\mathrm{TiO}_{2}$ as electrons and $\tau_{2}(3-4 \mathrm{~ns})$ was the component that relaxed to steady state in the dye [9].

Our fluorescence lifetime results are shown in Fig. 8b. $\tau_{1}$ did not change in relation to the particle size. Because $\tau_{1}$ had a short lifetime of approximately $0.3 \mathrm{~ns}$, it is thought that the contribution of this external factor was small. In contrast, $\tau_{2}$ decreased in IO356 and increased in IO457. To consider the contribution of photonic crystals to the change in this lifetime, the emission wavelength was compared with the photonic band diagram (Fig. 4b). In our system, the normalised frequency corresponding to the particle diameter in the first fluorescence wavelength peak $(\lambda=675 \mathrm{~nm})$ of Chlorine e6 was approximately $0.2,0.35,0.55$ and 0.8 for IO202, IO356, IO457 and IO731, respectively, i.e. in IO457, the fluorescent wavelength hits the quasi photonic band gap with a small 
slope in the normalised frequency of approximately 0.5. The state density of photon should be smaller near the band gap [51]. The low local density of photon states is generally causes low emission rate thus longer lifetime. It might be possible that the increased fluorescence lifetime was due to the photonic band. In addition, the error bars for $\tau_{1}$ and $\tau_{2}$ in IO457 were larger than those for other electrodes. The photonic band effect on a photon decreases because of the disorder of structure periodicity. Because a self-assembled photonic crystal includes random defects, the error bars in IO457 might have become large because of the random magnitude of the photonic band effects. On the other hand, on the normalised frequency of 0.35 corresponding to IO356, the dispersion relation showed monotone increase and the error bars in the fluorescence lifetime is small. It is expected that the contribution to the fluorescence lifetime by a photonic band was small. The reasons for the small $\tau_{2}$ in IO356 are expected to be factors other than photonic band.

\section{Conclusion}

We expected the fluorescence inhibition effect of the dye in a self-assembled photonic crystal. We prepared $\mathrm{TiO}_{2}$ inverse opals of various particle sizes by LPD and explored the effects of inverse opals on DSSCs from the perspective of structure, optical properties, electrochemical properties and fluorescence lifetime measurements in relation to the amount of adsorbed dye and compared with the photonic band diagram. Chlorine e6, a dye derived from chlorophyll, that has a long excited electron lifetime was used for this purpose.

A previous study of DSSCs incorporating an inverse opal primarily considered the harvesting effect of the incident light [12]. Miguez et al. [13] suggested that photoelectric conversion efficiency could be improved by utilising an inverse opal with a particle size of approximately $200 \mathrm{~nm}$. However, we found that IO356 exhibited the highest overall photoelectric conversion efficiency and the highest photoelectric conversion efficiency per one dye molecule. IO356 has low resistance, particularly for the diffusion resistance of the electrolyte and contact resistance between $\mathrm{TiO}_{2}$ interfaces. In addition, the higher internal quantum efficiency per one dye molecule and the incident light wavelength dependence were confirmed in IO457, in spite of the fact that the magnitude of the electrical resistance was almost the same as IO202. In IO457, because the extension of fluorescence lifetime by a photonic band was evident, it is possible that the photonic band influenced the internal quantum efficiency per one dye molecule. Here we should mention that, the normalized efficiency of IO457 was only about $60 \%$ of that of IO356. The effect on the photon-to-electron conversion efficiency per one molar dye seems less than the effect of the electric resistance in our system.
We consider that the differences between our results and those of previous researchers are because of the use of Chlorine e6, a naturally-occurring, chlorophyll derived dye that is known to have a long fluorescence lifetime. In addition, as evidenced by the excitation and fluorescence spectra of the dye, almost all absorbed light in a wide wavelength range was converted to a first peak emission (Supporting Information 3). Therefore, compared with the ruthenium dyes used by other researchers, our DSSC was susceptible to the structure of the photonic crystal. It should be noted that in $\mathrm{Yu}$ et al., the unexpectedly high scattering efficiency of the $450-\mathrm{nm} \mathrm{TiO} 2$ spheres was ascribed to the characteristic photonic reflection effect originating from the sphere's uniform size and long-range ordering using N719 dye adsorbed on submicron-sized monodispersed $\mathrm{TiO}_{2}$ spheres [34]. One possibility is that more excited electrons in the dye may have enabled the extension of the lifetime by the photonic band, which, in turn, might move to the conduction band of titanium oxide. As a result, the internal quantum efficiency per $1 \mathrm{~mol}$ of dye may have improved. A detailed verification of this assumption cannot be performed for a self-assembled photonic crystal with many defects. Such verification would require a dye sensitizing electrode with a full/complete photonic bandgap [52]. In future, we will also conduct the electron lifetime measurements in a DSSC and examine the results.

Acknowledgments The authors would like to thank Mr. Yohey Shibuya, Ms. Miho Kawai, Mr. Ryota Watanabe and Mr. Nobuhisa Hikichi for their valuable help. This work was partly supported by the Kazuchika Okura Memorial Foundation, Murata Science Foundation, and JSPS KAKENHI Grant Number 25420707.

Open Access This article is distributed under the terms of the Creative Commons Attribution License which permits any use, distribution, and reproduction in any medium, provided the original author(s) and the source are credited.

\section{References}

1. B. O'Regan, M. Gratzel, Nature 353(6346), 737-740 (1991)

2. K. Kalyanasundaram, Dye-sensitized solar cells (CRC Press, Lausanne, 2010)

3. A. Hagfeldt, G. Boschloo, L. Sun, L. Kloo, H. Pettersson, Chem. Rev. 110(11), 6595-6663 (2010)

4. A. Yella, H.-W. Lee, H.N. Tsao, C. Yi, A.K. Chandiran, M.K. Nazeeruddin, E.W.-G. Diau, C.-Y. Yeh, S.M. Zakeeruddin, M. Grätzel, Science 334(6056), 629-634 (2011)

5. Y. Liu, H. Lin, J.T. Dy, K. Tamaki, J. Nakazaki, D. Nakayama, S. Uchida, T. Kubo, H. Segawa, Chem. Commun. 47(13), 4010$4012(2011)$

6. Y. Hao, X. Yang, M. Zhou, J. Cong, X. Wang, A. Hagfeldt, L. Sun, ChemSusChem 4(11), 1601-1605 (2011)

7. J.-E. Moser, W.M.F. Lenzmann, M. Graetzel, Z. Phys. Chem. 212, 85-92 (1999) 
8. G. Calogero, G. Di Marco, S. Caramori, S. Cazzanti, R. Argazzi, C.A. Bignozzi, Energy Environ. Sci. 2(11), 1162 (2009)

9. A. Kay, R. Humphry-Baker, M. Graetzel, J. Phys. Chem. 98(3), 952-959 (1994)

10. S. Nishimura, N. Abrams, B.A. Lewis, L.I. Halaoui, T.E. Mallouk, K.D. Benkstein, J. van de Lagemaat, A.J. Frank, J. Am. Chem. Soc. 125(20), 6306-6310 (2003)

11. L.I. Halaoui, N.M. Abrams, T.E. Mallouk, J. Phys. Chem. B 109(13), 6334-6342 (2005)

12. S.-H.A. Lee, N.M. Abrams, P.G. Hoertz, G.D. Barber, L.I. Halaoui, T.E. Mallouk, J. Phys. Chem. B 112, 14415-14421 (2008)

13. A. Mihi, F.J. López-Alcaraz, H. Míguez, Appl. Phys. Lett. 88, 193110 (2006)

14. A. Mihi, H. Míguez, J. Phys. Chem. B 109(33), 15968-15976 (2005)

15. C.-A. Tao, W. Zhu, Q. An, G. Li, J. Phys. Chem. C 114(23), $10641(2010)$

16. L. Liu, S.K. Karuturi, L.T. Su, A.I.Y. Tok, Energy Environ. Sci. 4(1), 209-215 (2011)

17. S. Guldin, S. Hüttner, M. Kolle, M.E. Welland, P. Müller-Buschbaum, R.H. Friend, U. Steiner, N. Tétreault, Nano Lett. 10(7), 2303-2309 (2010)

18. E.S. Kwak, W. Lee, N.-G. Park, J. Kim, H. Lee, Adv. Funct. Mater. 19(7), 1093-1099 (2009)

19. J.-H. Shin, J.H. Moon, Langmuir 27(10), 6311-6315 (2011)

20. D.-J. Yang, S.-C. Yang, J.-M. Hong, H. Lee, I.-D. Kim, J. Electroceram. 24(3), 200-204 (2010)

21. J.T. Park, E.K. Roh, R. Patel, K.J. Son, W.-G. Koh, J.H. Kim, Electrochim. Acta 56(1), 68-73 (2010)

22. S.I. Matsushita, N. Fukuda, M. Shimomura, Colloids Surf. A 257-258, 15-17 (2005)

23. M. Shimomura, S. Matsushita, N. Fukuda, Japan (2005)

24. S. Matsushita, S. Fujikawa, S. Onoue, T. Kunitake, M. Shimomura, Bull. Chem. Soc. Jpn. 80(6), 1226-1228 (2007)

25. Y. Yagi, S.I. Matsushita, D.A. Tryk, T. Koda, A. Fujishima, Langmuir 16(3), 1180-1184 (2000)

26. S. Matsushita, C. Nishiyama, G. Kato, A. Nakajima, T. Isobe, T. Hashimoto, J. New Mat. Electr. Syst. 14, 229 (2011)

27. A. Buffaz, E. Oikawa, T. Hashimoto, S. Matsushita, J Nanosci Nanotecnol 9, 185-189 (2009)

28. T. Sawadaishi, M. Shimomura, Mol. Cryst. Liq. Cryst. 464, 227-231 (2007)

29. F. Sordello, V. Maurino, C. Minero, J. Mater. Chem. 21(47), 19144-19152 (2011)
30. H.T. Miyazaki, H. Miyazaki, K. Ohtaka, T. Sato, J. Appl. Phys. 87(10), 7152-7158 (2000)

31. S. Deki, Y. Aoi, O. Hiroi, A. Kajinami, Chem. Lett. 25, 433-434 (1996)

32. J.-H. Yum, E. Baranoff, F. Kessler, T. Moehl, S. Ahmad, T. Bessho, A. Marchioro, E. Ghadiri, J.-E. Moser, C. Yi, M.K. Nazeeruddin, M. Grätzel, Nat. Commun. 3, 631 (2012)

33. Z.-S. Wang, H. Kawauchi, T. Kashima, H. Arakawa, Coord. Chem. Rev. 248(13-14), 1381-1389 (2004)

34. I.G. Yu, Y.J. Kim, H.J. Kim, C. Lee, W.I. Lee, J. Mater. Chem. 21(2), 532-538 (2011)

35. S. Nakade, Y. Saito, W. Kubo, T. Kitamura, Y. Wada, S. Yanagida, J. Phys. Chem. B 107(33), 8607-8611 (2003)

36. G.K. Mor, K. Shankar, M. Paulose, O.K. Varghese, C.A. Grimes, Nano Lett. 6(2), 215-218 (2005)

37. M. Law, L.E. Greene, J.C. Johnson, R. Saykally, P. Yang, Nat. Mater. 4(6), 455-459 (2005)

38. J. Chae, M. Kang, J. Power Sour. 196(8), 4143-4151 (2011)

39. Q. Zhang, K. Park, J. Xi, D. Myers, G. Cao, Adv. Energy Mater. 1(6), 988-1001 (2011)

40. Y. Amao, J. Photochem. Photobiol. A 164(1-3), 47-51 (2004)

41. Q. Wang, J.-E. Moser, M. Grätzel, J. Phys. Chem. B 109(31), 14945-14953 (2005)

42. L. Han, N. Koide, Y. Chiba, T. Mitate, Appl. Phys. Lett. 84(13), 2433 (2004)

43. C. Longo, A.F. Nogueira, M.-A.D. Paoli, H. Cachet, J. Phys. Chem. B 106(23), 5925-5930 (2002)

44. T. Hoshikawa, M. Yamada, R. Kikuchi, K. Eguchi, J. Electrochem. Soc. 152(2), E68 (2005)

45. P.W. Barber, S.C. Hill, Light Scattering by Particles: Computational Methods (World Scientific, Singapore, 1990)

46. H.C. van de Hulst, Light Scattering by Small Particles (Dover, New York, 1981)

47. P.-E. Wolf, G. Maret, Phys. Rev. Lett. 55(24), 2696-2699 (1985)

48. J. Ferber, J. Luther, Sol. Energy Mater. Sol. Cells 54(1-4), 265-275 (1998)

49. J.-Y. Zhang, X.-Y. Wang, M. Xiao, Y.-H. Ye, Opt. Lett. 28(16), 1430-1432 (2003)

50. S. Kubo, A. Fujishima, O. Sato, H. Segawa, J. Phys. Chem. C 113(27), 11704-11711 (2009)

51. K. Inoue, M. Wada, K. Sakoda, A. Yamanaka, M. Hayashi, J.W. Haus, Jpn. J. Appl. Phys. 33(2), L1463-L1465 (1994)

52. S. Matsushita, O. Suavet, H. Hashiba, Electrochim. Acta 55(7), 2398-2403 (2010) 